\title{
Formation and Annealing Effect of Tolanethioacetate Self-Assembled Monolayers on Au(111)
}

\author{
Youngdo Jeong, Jin Wook Han, Nakjoong Kim, Youngil Lee, Changjin Lee, Masahiko Hara, and Jaegeun Noh ${ }^{\star}$ \\ Department of Chemistry, Hanyang University, Seonl 133-791, Korea. "E-mail:jg7toh@hamang.ac.kr \\ "Department of Chemistry, Ulsan University, Ulsan 680-749, Korea \\ *Advanced Material Division, Korea Research Institute of Chemical Technologv, Daejeon 305-600, Korea

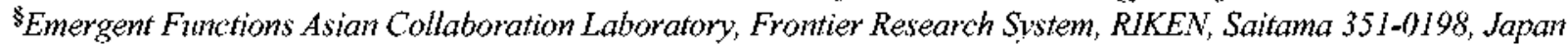 \\ Received September 18, 2007
}

\begin{abstract}
Self-assembled monolayers ( $\mathrm{S} \Lambda \mathrm{Ms}$ ) were formed by adsorption of thioacetyl-terminated tolanethioacetate (TT $\Lambda$ ) on $\Lambda \mathrm{u}(111)$ in a $0.5-\mathrm{mM}$ ethanol solution atter one day immersion at room temperature. Molecular-scale STM imaging revealed that the TT $\Lambda \mathrm{S} \Lambda \mathrm{M}$ s were composed of two mixed phases; an ordered phase with small domains describing a $(\sqrt{3} \times 2 \sqrt{3}) \mathrm{R} 30^{\circ}$ structure and a disordered phase. Interestingly, after annealing the precovered TT $\Lambda \mathrm{S} \Lambda \mathrm{M}$ s on $\Lambda \mathrm{u}(111)$ at $90^{\circ} \mathrm{C}$ for $1 \mathrm{~h}$, the small ordered domains grew unidirectionally, resulting in the formation of unique rod-like domains, which were assigned a $(\sqrt{3} \times 2 \sqrt{2}) R 7^{\circ}$ structure. These results will be very useful in understanding the formation and thermal behavior of TT $\Lambda$ S $\Lambda$ Ms on gold surfaces.
\end{abstract}

Key Words : Self-assembled monolayers, Tolanethioacetate, Ordered domains, Annealing effect, Scanning tunneling microscopy

\section{Introduction}

Self-assembled monolayers (SAMs) formed by organic thiols on metals have been extensively studied because of their fundamental interests and practical applications in adhesion, lubrication, corrosion, biosensors, nanolithography, and molecular electronics. ${ }^{1-9}$ Recently, tolane compounds containing a $\pi$-conjugated molecular backbone have drawn much attention in molecular electronics due to their intrinsic electronic properties, which can be tuned by varying the angle between two benzene rings in the molecule. ${ }^{10}$ However, it was discovered that the thiol group in aromatic thiols can be easily oxidized to disulfides or other oxidized compounds during SAM formation in a solution. ${ }^{11}$ To avoid oxidation, organic thioacetates protected by acetyl groups have been substituted for thiols for SAM fabrication. ${ }^{12,13}$ After deprotection of the acetyl group in acidic or basic solution, thiols or thiolates were formed, resulting in the formation of chemisorbed SAMs. Alternatively, if the SAMs had slightly different structural characteristics compared to SAMs prepared in acid- or base-catalyzed solution, the thioacetyl-terminated organic thioacetate on gold formed chemisorbed monolayers in a pure solvent. ${ }^{13-15}$

Structural control of SAMs formed on gold by conjugated organic molecules is a key issue for further application of SAM-based molecular electronics. It is well known that alkanethiol SAMs on gold have many ordered domains with clear domain boundaries and vacancy islands (VIs). ${ }^{16-18}$ For practical application of SAM-based devices, SAMs should contain large, ordered domains with a low density of VIs. Air or vacuum annealing of SAM samples at elevated temperatures is one of the best ways to increase the size of the ordered domains and reduce the number of VIs by reduction of the total step-edge energy. ${ }^{2.16 .17}$ It was also determined that a high quality of SAMs can be obtained by controlling the solution temperature, which largely affects the diffusion rate of the adsorbed molecules and gold atoms during the self-assembly process. ${ }^{19}$ As the solution temperature increases, the size of the ordered domains in SAMs on $\mathrm{Au}(111)$ increases, whereas the density of VIs decreases.

In this study, the 2D surface structure of thioacetylterminated tolanethioacetate (TTA) SAMs on Au(111) formed in $0.5 \mathrm{mM}$ ethanol solutions at room temperature were studied using scanning tunneling microscopy. The thermal annealing effect of pre-covered TTA SAMs on Au(111) was examined to elucidate the thermal stability of the SAMs at an elevated temperature. Molecular-scale STM images of TTA SAMs on Au(111) provided a better understanding of the formation and thermal stability, which considerably differs from organic thiol SAMs.

\section{Experimental Section}

Thioacetyl-terminated TTA compound containing $\pi$ conjugated phenyl groups was synthesized by modifying a previously reported method..$^{20}$ Figure 1 shows the structural formula of TTA molecule. As previously reported, Au(111) substrates were prepared by vacuum evaporation of gold on freshly cleaved mica sheets prebaked at $330^{\circ} \mathrm{C}$ for $2 \mathrm{~h}$ under a vacuum pressure of $10^{-7} \sim 10^{-8}$ Torr. $^{21}$ TTA SAMs on $\mathrm{Au}(111)$ were prepared by immersing the Au(111) substrates into a freshly prepared $0.5-\mathrm{mM}$ ethanol TTA solution at room temperature for one day (pre-covered SAMs). The SAM samples were removed from the solution and thoroughly rinsed with pure ethanol to remove physisorbed molecules from the SAM surface. To examine the thermal behavior, TTA SAMs were annealed at $90^{\circ} \mathrm{C}$ for $1 \mathrm{~h}$ in a nitrogen atmosphere. STM measurements were performed 


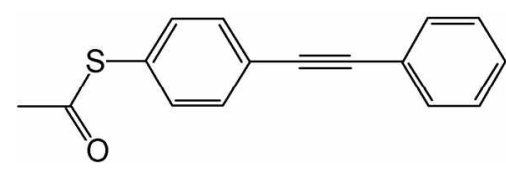

Figure 1. Structural formula of TTA molecule.

using a NanoScope E (Veeco, Santa Barbara, CA) with a commercial $\mathrm{Pt} / \mathrm{Ir}$ (80:20) tip. All STM images were obtained in air using the constant current mode with a bias voltage $\left(\mathrm{V}_{\mathrm{b}}\right.$ ) ranging from 200 to $700 \mathrm{mV}$ and a tunneling current $\left(\mathrm{I}_{\mathrm{b}}\right)$ between the tip and sample ranging from 0.15 to $0.50 \mathrm{nA}$. We note that all STM images are original without any filtering process, except the STM image of Figure 5a obtained after "low pass filter" treatment in NanoScope software.

\section{Results and Discussion}

The STM images in Figure 2 represent the surface structures of TTA SAMs on Au(I11) after one day immersion in a $0.5-\mathrm{mM}$ ethanol solution at room temperature. The STM image $(75 \mathrm{~nm} \times 75 \mathrm{~nm})$ in Figure $2 \mathrm{a}$ shows that TTA SAMs on $\mathrm{Au}(111)$ were composed of uneven monolayer phases with bright and dark regions. The STM image $(50 \mathrm{~nm} \times 50$ $\mathrm{nm}$ ) in Figure $2 \mathrm{~b}$ clearly shows the formation of $2 \mathrm{D}$ ordered phases (bright region) with small rod-like domains separated by disordered phases originating from the low surface coverage (dark region). The surface features of the TTA SAMs were considerably different from organic thiol SAMs consisting of long-range ordered domains, sharp domain boundaries, and many VIs. ${ }^{3,19}$ The dark regions in TTA SAMs have irregular shapes, which are largely different from those in organic SAMs that produce a round or triangular shape (referred to as VIs). The monatomic step height of VIs was approximately $2.5 \AA$. Figure $2 a^{\prime}$ shows the height profile along the line indicated on the STM image. The height
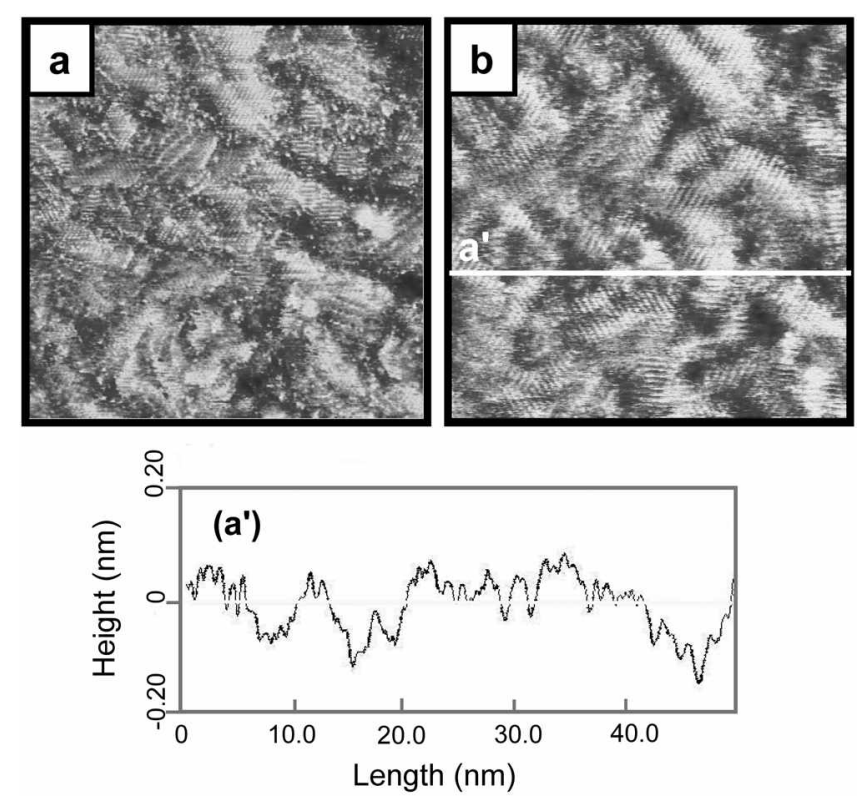

Figure 2. (a) and (b) STM images of TTA SAMs on Au(111) formed after 1 day immersion in a $0.5-\mathrm{mM}$ ethanol solution at room temperature (pre-covered TTA SAMs). Scan sizes were (a) $75 \mathrm{nun} \times 75 \mathrm{~nm}$ and (b) $50 \mathrm{~nm} \times 50 \mathrm{num}$, respectively. (a') show cross-sectional profile along line $\mathrm{a}^{\prime}$ on the STM image of (b).

values were in the range of 1.2 to $2.2 \AA$, which were different from the observed value of $2.5 \AA$ in the organic thiol SAMS. Therefore, the dark regions in the TTA SAMs were not VIs. The disordered phase appeared during phase transition between the "lying down" stripe phase and the "standing" closely packed phase. Alternatively, the adsorption activity of acetyl group-protected sulfur on gold was considered to be lower than sulfur on organic thiols due to the diminishing electron density of sulfur from the electronwithdrawing property of the acetyl group. As result, TTA
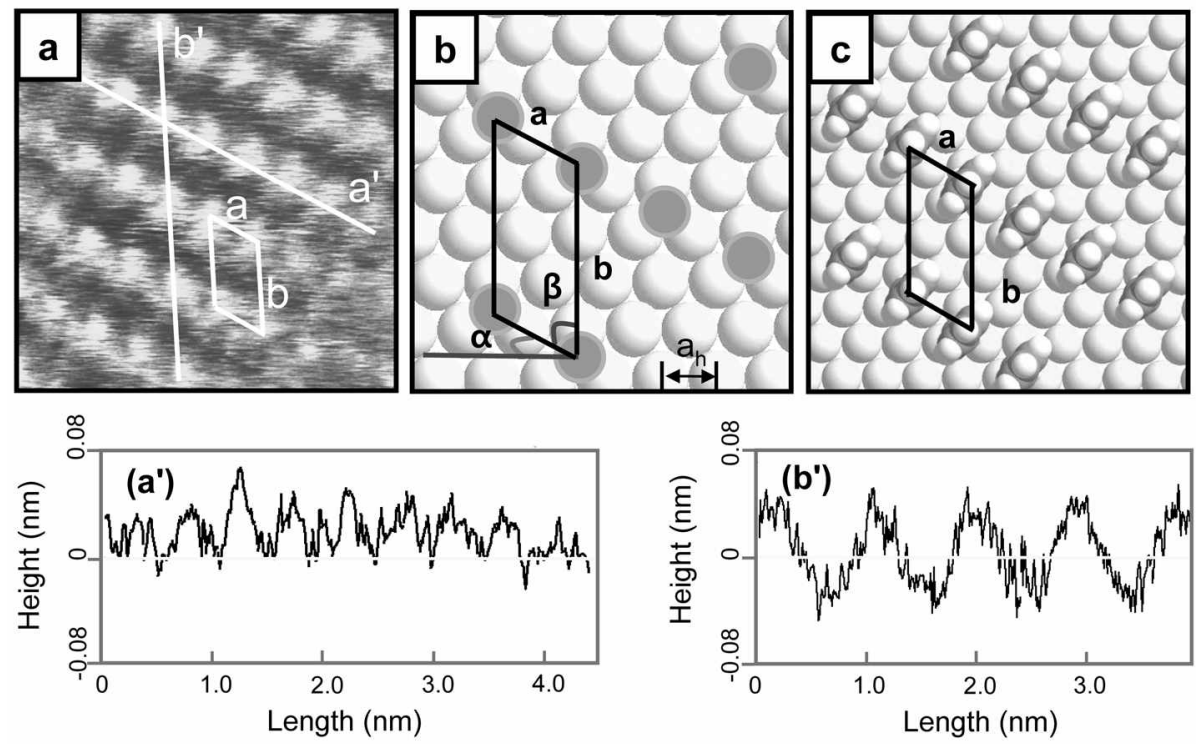

Figure 3. (a) High-resolution STM image $(4.5 \mathrm{~nm} \times 4.5 \mathrm{~nm}$ ) of TTA SAMs on Au(1111). Schematic (b) structural and (c) space-fill drawing models of the proposed TTA SAMs structure. $\left(a^{+}\right)$and $\left(b^{+}\right)$show cross-sectional profiles along lines $a^{+}$and $b^{\prime}$ on the STM image of (a), which indicate the high degree of structural order of TTA molecules on $\mathrm{AU}(111)$. 
molecules could slowly adsorb onto gold during selfassembly, which strongly affected the formation and 2D SAM growth. By comparing the domain structures of organic thiol and TTA SAMs on gold, it was reasonable to consider that the $2 \mathrm{D}$ ordered phases of TTA SAMs were formed via a kinetically controlled process. This assumption can be supported by the results that TTA SAMs with the uniform and large domains were obtained in ethanol solution containing tolanethiols made after deprotection of acetyl group via base catalyzed reaction (in $\mathrm{NH}_{4} \mathrm{OH}$ solution). This relationship will be reported in a separate paper.

The molecularly resolved STM image in Figure 3 a shows well-ordered $2 \mathrm{D}$ packing arrangements $(4.5 \mathrm{~nm} \times 4.5 \mathrm{~nm})$ of TTA SAMs on Au(111). The cross-sectional profiles in Figures $3\left(a^{\prime}\right)$ and $\left(b^{\prime}\right)$ along lines $a^{\prime}$ and $b^{\prime}$ corresponding to the oblique unit cell in Figure 3(a), show the periodic molecular arrangements of TTA molecules on Au(111). Based on the high-resolution STM image, lattice constants of an oblique unit cell were: $\mathrm{a}=5.0 \pm 0.1 \AA=\sqrt{3} \mathrm{ah}, \mathrm{b}=10.0 \pm$ $0.1 \AA=2 \sqrt{3} \mathrm{ah}, a=30 \pm 1^{\circ}$ and $\beta=60 \pm 1^{\circ}$ (see Figure $3 \mathrm{~b}$ ), where $\mathrm{a}_{\mathrm{h}}=2.89 \AA$ denotes the interatomic distance of the $\mathrm{Au}(111)$ lattice. Based on the STM image, a schematic structural model and space-fill drawing model for TTA SAMs adsorbed on $\mathrm{Au}(111)$ was proposed, as shown in Figures $3 \mathrm{~b}$ and $3 \mathrm{c}$. The molecular packing structure was assigned to a $(\sqrt{3} \times 2 \sqrt{3}) \mathrm{R} 30^{\circ}$ structure. From the model of Figure 3b, it was assumed that all the sulfur atoms in the TTA SAMs occupied three-fold hollow sites of the Au(I11) lattice. The average areal density of the adsorbed molecule was $50 \AA^{2} /$ molecule. The observed structure and average areal density of TTA SAMs formed were comparable to thioactyl-terminated oligo(phenylene ethynylene)s SAMs with a $(\sqrt{3} \times \sqrt{3}) \mathrm{R} 30^{\circ}$ structure and an average areal density of $21.6 \AA^{2} /$ molecule formed after deprotection of thioacetate group in acidic conditions. ${ }^{12}$ From these results,
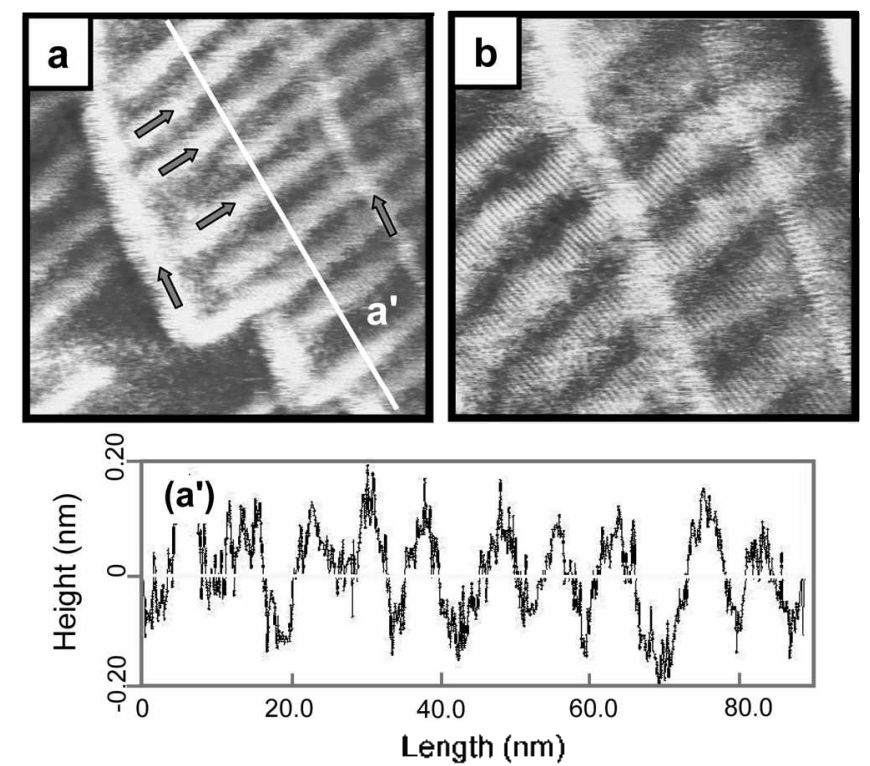

Figure 4. (a) and (b) STM images obtained after annealing the precovered TTA SAMs at $90^{\circ} \mathrm{C}$ for $1 \mathrm{~h}$. Scan sizes were (a) $75 \mathrm{~nm} \times$ $75 \mathrm{nun}$ and (b) $50 \mathrm{~nm} \times 50 \mathrm{nun}$, respectively. (a) show crosssectional profile along line a' on the STM image of (a).

it was determined the adsorption of thioacetyl-terminated TTA molecules on Au(111) in ethanol leads to the formation of $2 \mathrm{D}$ ordered SAMs with low surface coverage.

It is well known that thermal annealing in air or vacuum at an elevated temperature greatly enhances the SAM surface morphologies, such as by increasing the size of the ordered domains and reducing the number of VIs and structural defects. ${ }^{23}$ Specifically, thermal annealing can increase the diffusion rate of adsorbed molecules on a surface and the lateral diffusion of $\mathrm{Au}$ atoms from terrace to step edges, resulting in the formation of larger ordered domains and
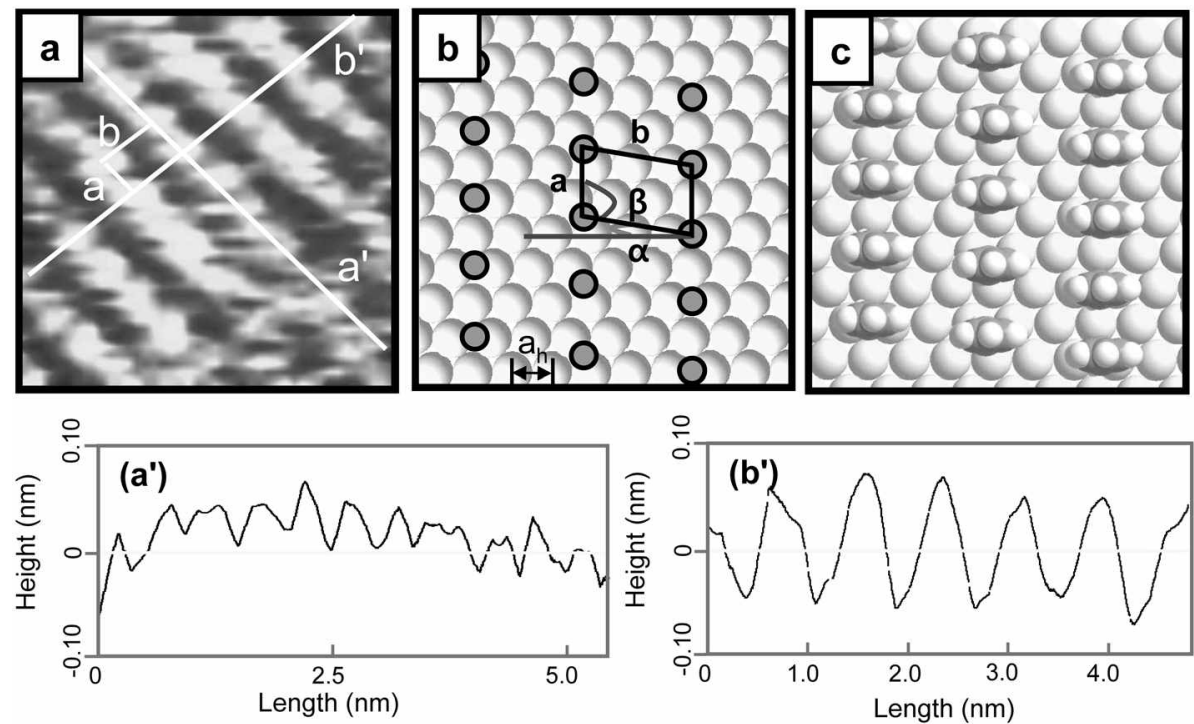

Figure 5. (a) High-resolution STM image ( $4 \mathrm{~nm} \times 4 \mathrm{~nm}$ ) of the annealed TTA SAMs on Au(1111). Note that this STM image was obtained after "low pass filter" treatment in NanoScope software. Schematic (b) structural and (c) space-fill drawing models of the proposed annealed TTA SAMs structure. ( $a^{+}$) and ( $b^{\prime}$ ) show cross-sectional profiles along lines $a^{+}$and $b^{\prime}$ on the STM inage of (a), which indicate the high degrec of structural order of TTA molceules on $\Lambda u(111)$. 
depletion of VIs. The STM images in Figure 4 show the surface structures of TTA SAMs on Au(111) after annealing at $90^{\circ} \mathrm{C}$ for $1 \mathrm{~h}$. Interestingly, as indicated by the arrows, the small ordered domains grew unidirectionally, resulting in the formation of rod-like ordered domains (Figure $4 \mathrm{a}, 75 \mathrm{~nm} \times$ $75 \mathrm{~nm})$. The STM image $(50 \mathrm{~nm} \times 50 \mathrm{~nm})$ in Figure $4 \mathrm{~b}$ clearly shows the ordered molecular rows in the directional ordered domains, which will be discussed later (see Figure 5). To date, the unidirectional $2 \mathrm{D}$ domain growth has not been observed in other thiol SAM systems. The crosssectional profile in Figure 4a' along line a' shows a 6-nm periodicity between ordered domains with the same topographic height. The periodicity is nearly similar to the 6.3 $\mathrm{nm}$ periodicity observed in the $(23 \times \sqrt{3})$ surface reconstruction of $\mathrm{Au}(111)$ surface. ${ }^{23.2+}$ Therefore, the direction of domain growth of TTA SAMs was considered to be guided by the structure of the gold surface reconstruction. It has been reported that the initial growth stage of striped phase domains by dioctadecyl sulfide on $\mathrm{Au}(111)$ were strongly influenced by gold surface reconstruction. ${ }^{21}$

The STM image $(4 \mathrm{~nm} \times 4 \mathrm{~nm})$ in Figure 5a shows the ordered packing arrangements of the annealed TTA SAMs on $\mathrm{Au}(111)$. The cross-sectional profiles in Figures 5a' and $5 b^{+}$along lines $a^{\prime}$ and $b^{+}$corresponding to the unit cell in Figure 5a show the periodicities of the adsorbed molecules on the TTA SAMs. Based on the STM observation, the lattice constants of an oblique unit cell were: $\mathrm{a}=5.0 \pm 0.1 \AA$ $=\sqrt{3} \mathrm{a}_{\mathrm{h}} \mathrm{b}=8.2 \pm 0.1 \AA=2 \sqrt{3} \mathrm{ah}, \alpha=7 \pm 1^{\circ}$ and $\beta=83 \pm 1^{\circ}$ (see Figure $5 \mathrm{~b}$ ), where $\mathrm{a}_{\mathrm{h}}=2.89 \AA$ denotes the interatomic distance of the Au(111) lattice. The packing structure was assigned as a $(\sqrt{3} \times 2 \sqrt{3}) \mathrm{R} 7^{\circ}$ structure. The schematic structural model and space-fill drawing model of the annealed TTA SAMs are shown in Figures $5 \mathrm{~b}$ and $5 \mathrm{c}$, respectively. From the model of Figure 5b, it is suggested that all sulfur atoms occupy bridge sites of the Au(111) lattice. From this result, it was determined the adsorption site conversion of sulfur atoms in TTA SAMs occurred from the three-fold hollow sites to the bridge sites. The bridge sites may be more energetically stable for the formation of high-density TTA SAMs. The average areal density of the adsorbed molecules was approximately $41 \AA^{2} /$ molecule, which revealed that the average areal density for TTA SAMs decreased from 50 to $41 \AA^{2} /$ molecule after annealing, indicating the increase of adsorption density in $2 \mathrm{D}$ packing arrangements.

\section{Conclusion}

Molecular-scale STM observation revealed that the adsorption of TTA molecules on $\mathrm{Au}(111)$ from a $0.5-\mathrm{mM}$ ethanol solution produces SAMs composed of two mixed phases: an ordered phase with small domains describing a $(\sqrt{3} \times 2 \sqrt{3}) \mathrm{R} 30^{\circ}$ structure and a disordered phase. After annealing the pre-covered SAMs on $\mathrm{Au}(111)$ at $90^{\circ} \mathrm{C}$ for 1 $\mathrm{h}$, the small ordered domains grew unidirectionally, resulting in the formation of unique rod-like domains, which can be described as a $(\sqrt{3} \times 2 \sqrt{2}) \mathrm{R}^{\circ}$ structure. In addition, after anmealing, the adsorption site transitioned from the threefold hollow sites to bridge sites.

Acknowledgement. This work was supported by the Korea Foundation for International Cooperation of Science \& Technology (KICOS) through a grant provided by the Korean Ministry of Science \& Technology (MOST) in No. $\mathrm{K} 20501000002-07-\mathrm{E} 0100$-00210. This work was also partially supported by Seoul R\&BD Program (10919).

\section{References}

I. Love, J. C.; Estroff, L. A.; Kriebel, J. K.; Nuzo, R. G.; Whitesides, G. M. Chem. Rev: 2005, 105, 1103.

2. Noh, J.; Jeong, Y.; Ito, E.; Hara, M. J. Phys. Chem. C 2007, $/ 1$, 2691 .

3. Noh, J.; Kalo, H. S.; Kawai, M.; Hara, M. J. Phys. Chem, B 2006, 110,2793 .

4. Noh, J.; Park, H.; Jeong, Y.; Kwon, S. Bull. Korean Chem. Soc. 2006, 27,403 .

5. Noh, J. Bull. Korean Chem. Soc. 2006, $27,944$.

6. Tour, J. M. Acc. Chem. Res. 2000, 33, 791.

7. Smalley, J. F.; Sachs, S. B.; Chidsey, C. E. D.; Dudek, S. P.; Sikes, H. D.; Creager, S. E.; Yu, C. J.; Feldberg, S. W.; Newlon, M. D. J. Am. Soc. Chem. 2004, 126, 14620 .

8. Flood, A. H.; Stoddart, J. F; Steuerman, D. W; Heath, J. R. Science 2004, 306, 2055.

9. Kagan, C. R.; Afzali, A.; Martek, R.; Gignac, L. M.; Solomon, P. M.; Schrou, A. G.; Ek, B. Nano Lett. 2003, 3, 119.

10. Chen, J.; Reed, M. A. Chem. Phys. 2002, 281, 127.

11. Cai, L.; Yao, Y.; Yang, J.; Price, D. W.; Tour, J. M. Chem. Mater. 2002, 14, 2905.

12. Stapleton, J. J.; Harder, P; Daniel, T. A.; Reinard, M. D.; Yao, Y.; Price, D. W.; Tour, J. M.; Allara, D. L. Langmitir 2003, 10, 8245.

13. Tour, J. M.; Jones II, L.; Pearson, D. L.; Lamba, J. J. S.; Burgin, T. P.; Whitesides, G. M.; Allara, D. L.; Parikh, A. N.; Atre, S. V. J. Am. Chem. Soc. 1995, 117, 9529.

14. Kang, Y.; Won, D.; Kim, S. R.; Seo, K.; Choi, H.-S.; Lee, G.; Noh, Z.; Lee, T. S.; Lee, C. Mater. Sci. Eng. C 2004, 24, 43.

15. Jeong, Y; Lee, C.; Ito, E.; Hara, M.; Noh, J. Jpn. J. Appl. Phys. $2006,45,5906$.

16. Bucher, J.-P.; Santesson, L.; Kern, K. Langmirir 1994, 10,979.

17. Sondag-Huethorsı, J. A. M.; Schönenberger, C.; Fokkink, L. G. J. J. Phys. Chem. 1994, 98, 6826 .

18. Schönenberger, C.; Sondag-Huethorst, J. A. M.; Jorritsma, J.; Fokkink, L. G. J. Langmuir 1994, 10,611.

19. Yamada, R.; Wano, H.; Uosaki, K. Langmuir 2000, 16, 5223.

20. Huang, S. H.; Tour, J. M. J. Org. Chem. 1999, 64, 8898.

21. Noh, J.; Murase, T.; Nakajima, K.; Lee, H.; Hara, M. J. Phys. Chemi, B 2000, 110, $741 \mathrm{l}$.

22. Yang, G.; Liu, G.-y. J. Phys. Chen. B 2003, $107,8746$.

23. Chambliss, D. D.; Willson, R. J.; Chiang, S. Phys. Rev. Letf. 1991, 66,1761 .

24. Chambliss, D. D.; Willson, R. J. J. Vac. Sci. Technol. B 1991, 9, 933. 